\title{
Potential Mechanisms Underlying the Deleterious Effects of Synthetic Cannabinoids Found in Spice/K2 Products
}

\author{
Balapal S. Basavarajappa ${ }^{1,2,3,4, *(\mathbb{D})}$ and Shivakumar Subbanna ${ }^{1}$ \\ 1 Division of Analytical Psychopharmacology, Nathan Kline Institute for Psychiatric Research, Orangeburg, \\ NY 10962, USA; Subbanna.Shivakumar@nki.rfmh.org \\ 2 New York State Psychiatric Institute, New York, NY 10032, USA \\ 3 Department of Psychiatry, College of Physicians\& Surgeons, Columbia University, New York, NY 10032, USA \\ 4 Department of Psychiatry, New York University Langone Medical Center, New York, NY 10016, USA \\ * Correspondence: Basavaraj.Balapal@nki.rfmh.org; Tel.: +1-845-398-3234; Fax: +1-845-398-5451
}

Received: 27 December 2018; Accepted: 13 January 2019; Published: 16 January 2019

\begin{abstract}
The chief psychoactive constituent of many bioactive phytocannabinoids ( $\Delta^{9}$-tetrahydrocannabinol, $\Delta^{9}$-THC) found in hemp, cannabis or marijuana plants are scientifically denoted by the Latin term, Cannabis sativa, acts on cell surface receptors. These receptors are ubiquitously expressed. To date, two cannabinoid receptors have been cloned and characterized. Cannabinoid receptor type 1 (CB1R) is found to serve as the archetype for cannabinoid action in the brain. They have attracted wide interest as the mediator of all psychoactive properties of exogenous and endogenous cannabinoids and they are abundantly expressed on most inhibitory and excitatory neurons. Recent evidence established that cannabinoid receptor type 2 (CB2R) is also expressed in the neurons at both presynaptic and postsynaptic terminals and are involved in neuropsychiatric effects. Distinct types of cells in many regions in the brain express CB2Rs and the cellular origin of CB2Rs that induce specific behavioral effects are emerging. To mimic the bliss effects of marijuana, synthetic cannabinoids (SCBs) have been sprayed onto plant material, and this plant material has been consequently packaged and sold under brand name "Spice" or "K2". These SCBs have been shown to maintain their affinity and functional activity for CB1R and CB2R and have been shown to cause severe harmful effects when compared to the effects of $\Delta^{9}$-THC. The present review discusses the potential brain mechanisms that are involved in the deleterious effects of SCBs.
\end{abstract}

Keywords: marijuana; cannabinoid receptors; K2; Spice; gene expression; CREB

\section{Introduction}

Marijuana is the major often abused illegal drug (Figure 1). The degree of abuse raised from 14.5 million in 2007 to 18.1 million in 2011, with a projected about 5 million adult daily cannabis abusers. Marijuana abuse appears to begin in the eighth grade in 1.3\% of children and between 12 and 17 years old in $7.9 \%$ of children [1]. Besides, the typical content of $\Delta^{9}$-tetrahydrocannabinol ( $\left.\Delta^{9}-\mathrm{THC}\right)$, which is a major psychoactive component of many bioactive cannabinoids established in the Cannabis sativa plant [2], in marijuana has also doubled from 3.4\% in 1993 to $8.8 \%$ in 2008 [3]. Moreover, an upsurge in the concentration of $\Delta^{9}$-THC (13.8\%) in highly potent plant varieties (sinsemilla, 'skunk') [3] was also found in this period. The psychological effects that were detected after $\Delta^{9}$-THC use are comparable to those observed after recreationally consumed cannabis [4]. The majority of $\Delta^{9}$-THC effects are exerted via the endocannabinoid (EC) system. The EC system comprises receptors for $\Delta^{9}$-THC known as cannabinoid receptors type 1 and 2 (CB1R and CB2R, respectively), endogenous receptor ligands ('endocannabinoids', ECs), and EC synthesizing and degrading enzymes [5]. CB1R is ubiquitously 
expressed in brain regions, such as the hippocampus, basal ganglia, cortex, amygdala, and cerebellum, all of which are areas connected with the behavioral effects of $\Delta^{9}$-THC [6]. The EC system has a homeostatic role, but its dysfunction can promote pathological conditions $[7,8]$.

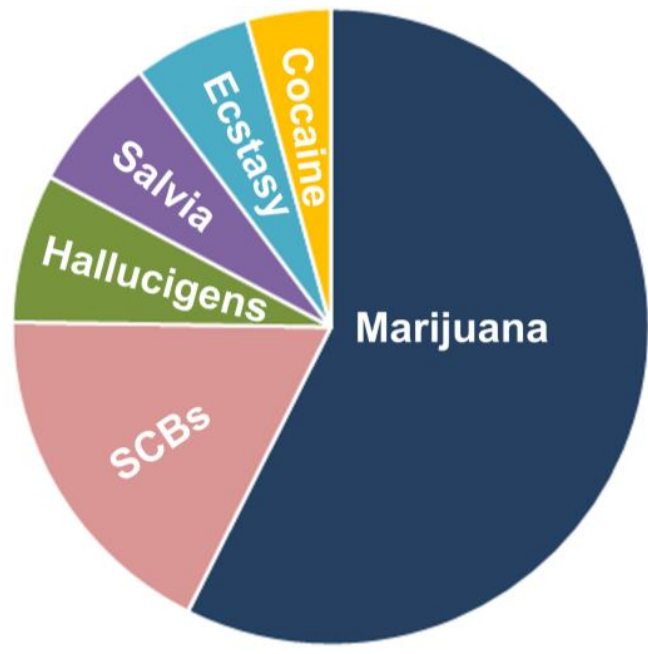

Figure 1. Pie illustration outlining the synthetic cannabinoid (SCB) abuse rate among high school-going children. SCB abuse is the most widespread among young people; of the illicit drugs most used by high school seniors, the use of SCBs are second only to that of marijuana (http://www.drugabuse.gov/publications/drugfacts/spice-synthetic-marijuana). The most common reasons for using SCBs were affordability, inability to detect SCBs in standard drug tests, and perceived physical and emotional benefits.

The CB1Rs and CB2Rs belong to the large superfamily of heptahelical G protein-coupled receptors (GPCR) and couple with $\mathrm{G}_{\mathrm{i}} / \mathrm{o}$ proteins (for more details, see reviews [5,7]). CB1R is one of the highly abundant GPCRs in the brain, with densities that are similar to the levels of $\gamma$-aminobutyric acid (GABA)- and glutamate-gated ion channels [9]. Functional CB2R is also present in limited amounts and distinct locations in the brains of several animal species, including humans [10]. The existence of CB2R in the brain has been acknowledged in distinct locations of the CNS in many animal species, including humans, in moderate levels, and is restricted to microglia and vascular elements [11]. However, the specific functions of this receptor in the CNS are emerging slowly. Recent strong evidence suggests the presence of CB2R mRNA in neuronal cells of the hippocampus [12] and dopamine-expressing neurons in the ventral tegmental area (VTA) $[13,14]$. CB2R-mediated regulation of cell type-specific synaptic plasticity was shown in the hippocampus [15-17]. Furthermore, increased CB2R levels in neurons were noticed under pathological conditions [18,19]. The selective agonists and antagonists of CB1R and CB2R are shown in Figures 2 and 3.

The stimulation of CB1R promotes its interaction with G-proteins, resulting in guanosine diphosphate/guanosine triphosphate exchange and the subsequent dissociation of the $\alpha$ and $\beta \gamma$ subunits. These subunits regulate the activity of multiple downstream effector proteins to produce biological functions. CB1Rs are coupled with $G_{i}$ or $G_{0}$ proteins. However, their affinity for $G_{i}$ or $G_{0}$ proteins might vary, as revealed by several receptor ligands and receptor ligand-stimulated GTP $\gamma$ S-binding studies [20]. CB1R activity differs from several other GPCR-G proteins, as it is precoupled with G-proteins and it is hence constitutively functional in the absence of exogenous agonists [21]. The CB-mediated signal transduction pathway is shown in Figure 4. Activation of CB1R by R-(+)-methanandamide (MetAEA) and ECs in N18TG2 cells inhibited adenylate cyclase (AC) activity (for review see [7]). In certain conditions, the enhanced AC activity was reported without $G_{i} / o$ coupling (pertussis toxin-sensitive), probably through the stimulation of $\mathrm{G}_{\mathrm{s}}$ proteins [22]. In vitro experiments, the expression of specific isoforms of AC (I, III, V, VI, or VIII) with the coexpression of CB1R was shown to inhibit cyclic adenosine monophosphate (cAMP) accumulation. However, the expression of II, IV, or 
VII AC isoforms, along with the CB1R coexpression was shown to enhance cAMP accumulation [23]. Interestingly, whether the coupling of CB1R with $\mathrm{G}_{\mathrm{s}}$ proteins has physiological function and whether this coupling enhances after $G_{i}$ or $G_{o}$ protein removal through colocalized noncannabinoid $G_{i} / o$ protein-coupled receptors have yet to be investigated. Further studies of the mechanism through which CB1R stimulation primes to the buildup of G $\alpha \mathrm{GTP} \beta \gamma$ heterotrimers would improve our knowledge on the CB1R mediated signal transduction mechanisms. It is also imperative to establish whether these heterotrimers $(\mathrm{G} \alpha, \mathrm{G} \beta \gamma$, and $\mathrm{G} \alpha \mathrm{GTP} \beta \gamma)$ can cooperate with distinct downstream effector targets to afford specificity to the signaling pathways.

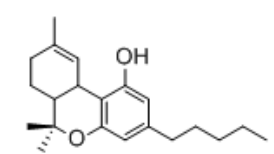

$\Delta^{9}$-Tetrahyrocannabinol

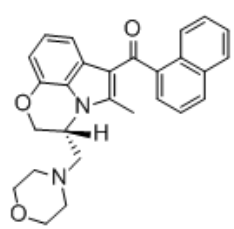

R(+)-WIN 55,212-2

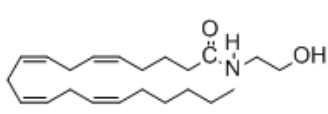

Anandamide

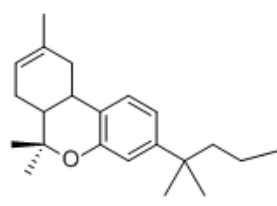

JWH133

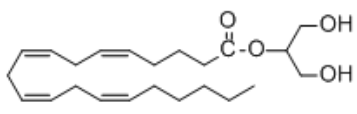

2-Arachidonoylglycerol<smiles>CCCn1c(C)c(C(=O)c2ccc3ccccc3c2)c2ccccc21</smiles>

JWH015

Figure 2. The structure of natural, endogenous and selective synthetic cannabinoid agonists of Cannabinoid receptors type 1 and type 2 (CB1R and CB2R).

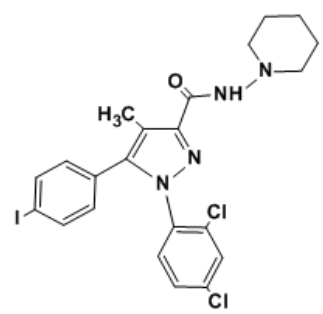

AM251

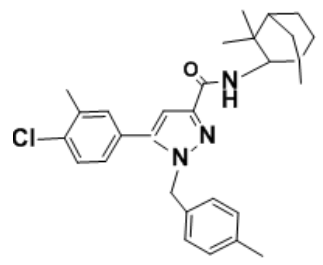

SR144528

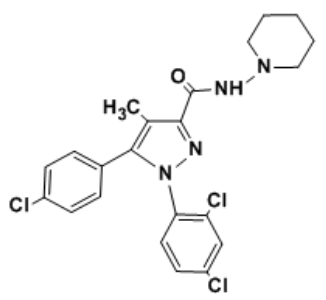

SR141716A<smiles>COc1ccc(C(=O)c2c(C)n(CCN3CCOCC3)c3cc(I)ccc23)cc1</smiles>

AM630

Figure 3. The structure of selective CB1R and CB2R antagonists. 


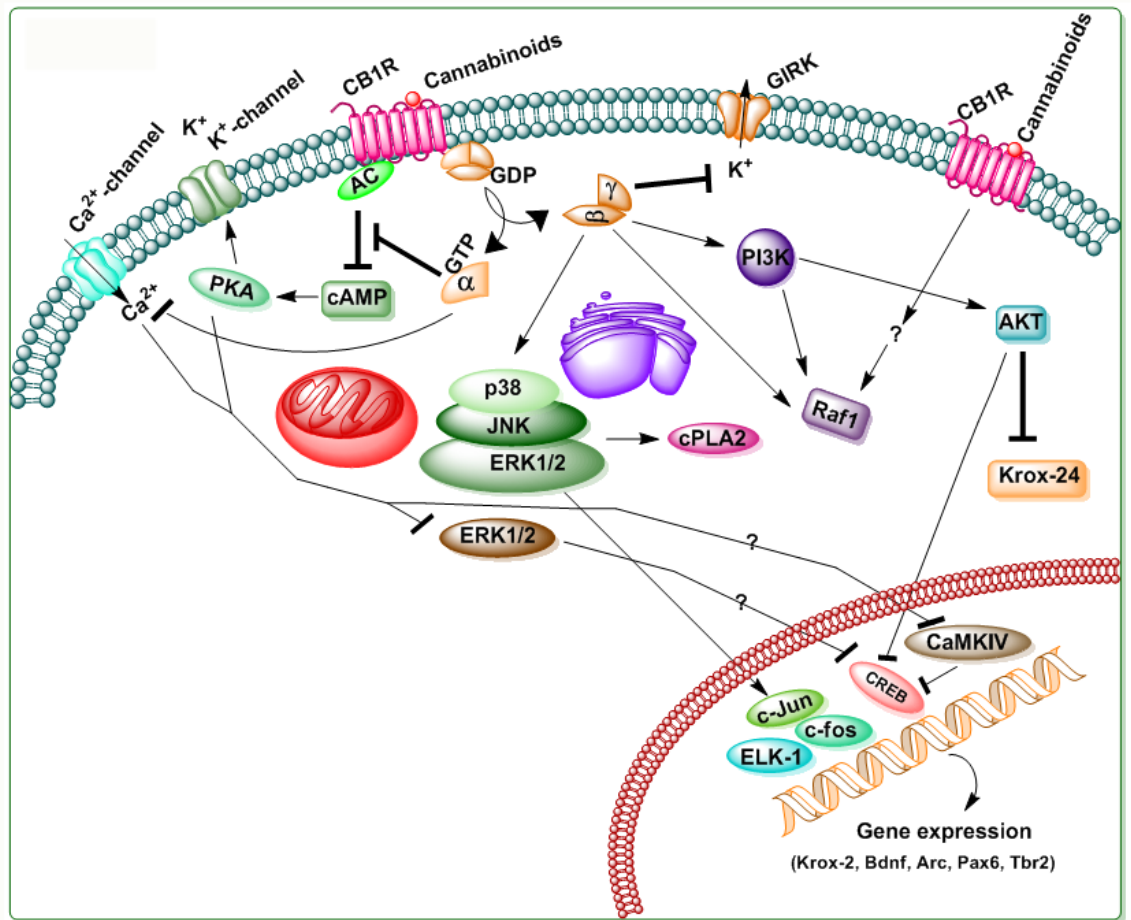

Figure 4. Schematic illustration of the CB1R mediated signaling. $\Delta^{9}$-tetrahydrocannabinol $\left(\Delta^{9}-\mathrm{THC}\right)$ and other SCBs produce their effects via binding to CB1Rs, a 7-transmembrane domain G-protein coupled receptor located in the cell membrane. The $\mathrm{Ca}^{2+}$ channels inhibited by CB1R stimulation include N-, P/Q-, and L-type channels. The actions on $\mathrm{Ca}^{2+}$ channels and adenylyl cyclase (AC) are thought to be mediated by the $\alpha$ subunits of the G-protein, and the effects on $\mathrm{G}$ protein-coupled inward rectifying $\mathrm{K}^{+}$(GIRK) and PI3K are considered to be mediated by the $\beta \gamma$ subunits. AC inhibition and the subsequent decrease in cyclic adenosine monophosphate (cAMP) lead to the inhibition of cAMP-dependent protein kinase (PKA). This leads to decreased $\mathrm{K}^{+}$channels and pCaMIV and pCREB levels, which might lead to the inhibition of gene expression required for several physiological functions. Stimulatory effects are indicated by $(\rightarrow)$ symbols and inhibitory effects by $(\perp)$ symbols.

CB2R belongs to the seven-transmembrane domain class of GPCRs. CB2R is also coupled to $G_{i} / o$ proteins; thus, the stimulation of $C B 2 R$ is associated with the inhibition of $A C$ and the cAMP/PKA-dependent pathway, as has been observed for CB1R. CB2R stimulation activates MAPK cascades, specifically the ERK and p38 MAPK cascades [24]. Additionally, the activation of CB2R has also been linked to the stimulation of additional intracellular pathways, including the PI3K/Akt pathway [24] (Figure 5). These pathways have been associated with pro-survival effects, as well as with the de novo synthesis of the sphingolipid messenger ceramide [24], which has been linked to the pro-apoptotic effects of CBs. CB2R activation by the selective agonist AM1241 contributes to the regeneration of DA neurons through increased activation of the PI3K/AKT signaling pathway following MPTP-induced neurotoxicity in mice [25]. Activation of CB2R by JWH133 induced neuroinflammation by enhancing the expression of MAPK phosphatase-1 (MKP-1), followed by the inhibition of MAPK signaling and increased blood-brain barrier permeability in a rat model of intracerebral hemorrhage (ICH) [26]. The activation of CB2R is also associated with the activation of CREB, followed by increased expression of Bcl-2, an anti-apoptotic gene leading to decreased cleaved caspase-3 levels and augmented neurological deficits induced by subarachnoid hemorrhage (SAH) in male rats [27]. The activation of CB2R has been shown to increase $G$ protein-coupled inward rectifying $\mathrm{K}^{+}$(GIRK) channel activity in pyramidal cells [28]. The activation of CB2R regulates the function of the sodium-bicarbonate cotransporter, causing a hyperpolarization of the neuron in a self-regulatory manner, independent of CB1R activity. These results provide additional evidence for the neuronal expression of CB2Rs and underscore the function of CB2R in neuronal transmission [17]. 


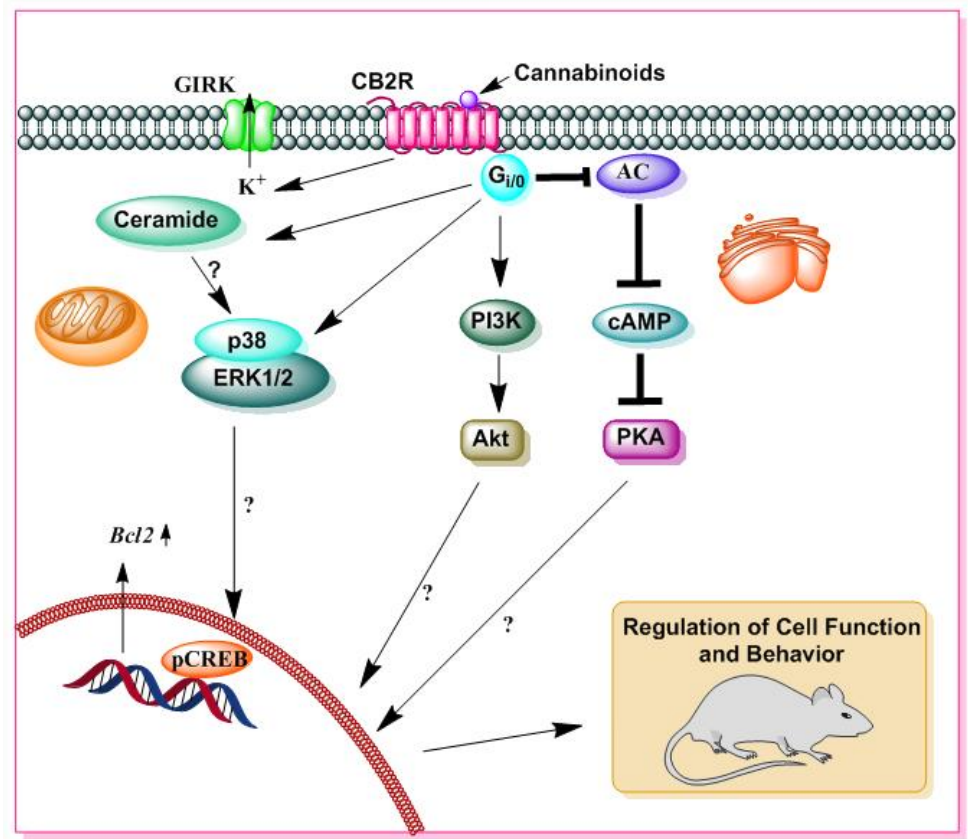

Figure 5. A schematic diagram of CB2R mediated signaling. $\Delta^{9}-\mathrm{THC}$ and SCBs also bind to CB2R. Similar to CB1R, CB2R is also a 7-transmembrane domain G-protein coupled receptor that is located in the cell membrane. The activation of CB2R is associated with several distinct cellular processes, such as GIRK, adenylate cyclase (AC), cAMP, PKA, ERK, p38 MAPK, and Akt pathways, as well as the pathway for the de novo synthesis of ceramide. Stimulatory effects are represented by $(\rightarrow)$ symbols and inhibitory effects by $(\perp)$ symbols.

SCBs were synthesized as research tools to investigate the function of the EC system [29]. However, within the last decade, SCBs have been sprayed onto plant material, which has then been packaged and sold as "Spice" or "K2". SBCs are also sold as liquids that can be vaporized and inhaled via e-cigarettes or other devices. These products are also identified as herbal or liquid incenses. These products mimic the effects of marijuana [30], because SCBs act on the same CB receptors as $\Delta^{9}$-THC, the psychoactive component in marijuana. Although these packages are labeled "not for human consumption", these products are often smoked, resulting in a marijuana-like high, as well as other physiological effects. These products are often marketed as safe, legal alternatives to marijuana. They are dangerous and may affect the brain much more strongly than marijuana; their particular effects can be variable and, in some cases, fatal. Many synthetic cannabinoids (SCBs) are now classified as Schedule I drugs under the United States (U.S.) Controlled Substances Act [31]. Because SCBs are now illegal and are classified in the most dangerous category of scheduled drugs, manufacturers have attempted to circumvent these laws by changing the more structurally diverse cannabimimetic compounds in their mixtures, which might not be listed under scheduled drug regulations. In addition, "medical" marijuana is being legalized in many states across the U.S. Therefore, individuals who previously would not have risked the consequences of procuring an illegal drug might now consider exposing themselves to marijuana and SCBs. In addition, the legal availability of marijuana may decrease use of SCB in the future.

SCBs were classified (Table 1) based on the chemical structures of the molecules [32]. The pharmacological effects of many SCBs vary widely and have been recently reviewed [33]. The most frequently investigated SCBs are the aminoalkylindole WIN55,212-2, the cyclohexylphenol CP55,940, and HU-210. Only a few studies have examined the naphthoylindoles (JWH-018 and analogs) or the newer synthetic structural families of cannabinoids that are now dominating the "Spice" market [33]. SCBs and their metabolites have been found to possess a higher binding affinity for CB receptors than $\Delta^{9}$-THC, which implies greater potency, greater harmful effects, and perhaps a longer duration of action. Although most Spice cannabinoids are widely known to be potent CB1R agonists, knowledge of 
the exact mechanisms that underlie their severe deleterious effects is largely limited. In this review, the authors provide an integrative overview of the current research on the mechanisms that are involved in the detrimental effects of Spice compounds.

Table 1. The Classification of Cannabinoids (CBs) [32].

\begin{tabular}{cc}
\hline \multicolumn{1}{c}{ Class } & Examples \\
\hline Classical cannabinoids & $\Delta^{9}$-THC, HU-210, AM-906, AM-411, O-1184. \\
\hline Non-classical cannabinoids & CP-47,497-C8, CP-55,940, CP-55,244. \\
\hline Hybrid cannabinoids & AM-4030. \\
\hline Aminoalkylindoles & $\begin{array}{c}\text { JWH-018, JWH-073, JWH-398, JWH-015, JWH-122, JWH-210, JWH-081, } \\
\text { JWH-200, WIN-55,212, JWH-250, JWH-251, Pravadoline, AM-694, RSC-4. }\end{array}$ \\
\hline Eicosanoids & Anandamide and methanandamide. \\
\hline Others & $\begin{array}{r}\text { Diarylpyrazoles (SR141716A), naphtoylpyrroles (JWH-307), } \\
\text { naphthylmethylindenes or derivatives of }\end{array}$ \\
& naphthalene-1-yl-(4-pentyloxynaphthalen-1-yl) methanone (CRA-13). \\
\hline
\end{tabular}

Spice products comprise several SCBs, and their potency at CB1R and CB2R differ. Therefore, their signaling mechanisms may also vary. Recently, a list of SCBs detected in Spice products, as well as the effects of these cannabinoids, was reviewed [33]. Some known physiological effects of SCBs are listed in Table 2. SCBs with a higher affinity for either CB1R or CB2R were shown to elicit adverse neurobehavioral effects. The majority of SCBs found in Spice products (Table 3), such as AM5983, AM678, AM2233, AM2389, SDB-001, AM4054, UR-144, XLR-11, JWH-081, and JWH-073, were shown to have higher affinity for CB1R than CB2R; these SCBs are expected to be similar to $\Delta^{9}$-THC in their action and suggested to be more severe than $\Delta^{9}-\mathrm{THC}$, with some of them displaying other symptoms [34]. A few SCBs found in Spice products, such as JWH-018, AM1710, and JWH-133, shown to have a higher affinity for CB2R than for CB1R (for references, see (https://www.drugs-forum.com/forum/showthread.php?t=117873)). Selected CB1R and CB2R agonists appear to bind off target sites, such as sodium channels, $\mu$ and $\delta$ opioid receptors, muscarinic acetylcholine receptors, $5-\mathrm{HT}_{3}$ receptors, and glycine receptors (for more details see [35]). Although details are limited, some designer drugs that are found in spice products have been shown to exert their action through non-CB1R and CB2R targets [36-38]. However, the mechanisms by which SCBs instigate their potentially harmful effects are not well established.

Table 2. Some known physiological effects of synthetic cannabinoids (SCBs) [33].

\begin{tabular}{c}
\hline Raised heart rate \& blood pressure \\
\hline Altered state of consciousness \\
\hline Mild euphoria and relaxation \\
\hline Perceptual alterations (time distortion) \\
\hline Intensification of sensory experiences \\
\hline Pronounced cognitive effects \\
\hline Impaired short-term memory \\
\hline Agitation, seizures, hypertension, emesis and hypokalemia \\
Increase in reaction times
\end{tabular}


Table 3. Effects of SCBs identified in Spice/K products.

\begin{tabular}{|c|c|c|c|c|c|c|}
\hline SPCs & Dose & Animal & Exposure & Parameter & Effects & Reference \\
\hline \multirow{11}{*}{ JWH-018 } & $3 \mathrm{mg} / \mathrm{kg}$ (i.p.) & Mouse & Acute & Cannabinoid tetrad & $>\Delta^{9}-\mathrm{THC}$ & [39] \\
\hline & $0.032 \mathrm{mg} / \mathrm{kg}$ (i.v.) & Monkey & Acute & Drug discrimination & $>\Delta^{9}-\mathrm{THC}$ & [40] \\
\hline & $3 \mathrm{mg} / \mathrm{kg}$ (i.p.) & Rat & Acute & Drug discrimination & $\begin{array}{c}>\text { AM5983 } \\
>\text { AM2233 } \\
\text { > WIN 55, 212-2 } \\
>\Delta^{9}-\text { THC }\end{array}$ & [41] \\
\hline & $2.5 \mathrm{mg} / \mathrm{kg}$ (i.p.) & Rat & Acute & Locomotion and catalepsy & $>\Delta^{9}-\mathrm{THC}$ & [42] \\
\hline & $\begin{array}{c}5.8 \% \text { (10-50 mg plant } \\
\text { material), inhale }\end{array}$ & Mouse & Acute & Cannabinoid tetrad & $>\Delta^{9}-\mathrm{THC}$ & [43] \\
\hline & $0.1-1.8 \mu \mathrm{mol} / \mathrm{kg}$ (i.v.) & Mouse & Acute & Cannabinoid tetrad & $>\Delta^{9}-\mathrm{THC}$ & [44] \\
\hline & $0.03-0.3 \mathrm{mg} / \mathrm{kg}$ (i.p.) & Mouse & Acute & Cannabinoid tetrad & Induced & [45] \\
\hline & $10 \mathrm{mg} / \mathrm{kg}$ (i.p.) & Mice & Acute & convulsion & Induced & [46] \\
\hline & 2 and $3 \mathrm{mg} / \mathrm{kg}$ (vapor) & Human & Acute & $\begin{array}{l}\text { neurocognitive function and } \\
\text { subjective feelings }\end{array}$ & Impaired & [47] \\
\hline & 1 and $100 \mathrm{nM}$ & $\begin{array}{l}\text { HP } \\
\text { Neuron }\end{array}$ & Acute & mEPSC frequency & Reduced & [48] \\
\hline & $5 \mathrm{nM}-5 \mu \mathrm{M}$ & Mouse brain slice & Acute & fEPSP & Impaired & [49] \\
\hline $\begin{array}{l}\text { JWH018 4-hydroxyindole } \\
\text { metabolite }\end{array}$ & 10 mg/kg (i.p.) & Mouse & Acute & Cannabinoid tetrad & $>\Delta^{9}-\mathrm{THC}$ & [39] \\
\hline JWH-167 & $0.1-6.0 \mu \mathrm{mol} / \mathrm{kg}$ (i.v.) & Mouse & Acute & Cannabinoid tetrad & $>\Delta^{9}-\mathrm{THC}$ & [44] \\
\hline JWH-203 & $0.13-13 \mu \mathrm{mol} / \mathrm{kg}$ (i.v.) & Mouse & Acute & Cannabinoid tetrad & $>\Delta^{9}-\mathrm{THC}$ & [44] \\
\hline JWH-204 & $0.8-2 \mu \mathrm{mol} / \mathrm{kg}$ (i.v.) & Mouse & Acute & Cannabinoid tetrad & $>\Delta^{9}-\mathrm{THC}$ & [44] \\
\hline JWH-205 & $13-19 \mu \mathrm{mol} / \mathrm{kg}$ (i.v.) & Mouse & Acute & Cannabinoid tetrad & $>\Delta^{9}-\mathrm{THC}$ & [44] \\
\hline JWH-251 & $0.9-6 \mu \mathrm{mol} / \mathrm{kg}$ (i.v.) & Mouse & Acute & Cannabinoid tetrad & $>\Delta^{9}-\mathrm{THC}$ & [44] \\
\hline JWH-208 & $2.8-38 \mu \mathrm{mol} / \mathrm{kg}$ (i.v.) & Mouse & Acute & Cannabinoid tetrad & $>\Delta^{9}-\mathrm{THC}$ & [44] \\
\hline JWH-237 & $1.5-3.0 \mu \mathrm{mol} / \mathrm{kg}$ (i.v.) & Mouse & Acute & Cannabinoid tetrad & $>\Delta^{9}-\mathrm{THC}$ & [44] \\
\hline JWH-306 & $1.1-2.9 \mu \mathrm{mol} / \mathrm{kg}$ (i.v.) & Mouse & Acute & Cannabinoid tetrad & $>\Delta^{9}-\mathrm{THC}$ & [44] \\
\hline AM2389 & $0.1-0.3 \mathrm{mg} / \mathrm{kg}$ (i.p.) & Rat & Acute & $\begin{array}{c}\text { Hypothermia and Drug } \\
\text { discrimination }\end{array}$ & $\begin{array}{l}>\text { AM5983 } \\
>\Delta^{9}-\mathrm{THC}\end{array}$ & [50] \\
\hline
\end{tabular}


Table 3. Cont

\begin{tabular}{|c|c|c|c|c|c|c|}
\hline SPCs & Dose & Animal & Exposure & Parameter & Effects & Reference \\
\hline AM5983 & $3 \mathrm{mg} / \mathrm{kg}$ (i.p.) & Rat & Acute & Drug discrimination & $\begin{array}{c}>\text { JWH-018 } \\
>\text { AM2233 } \\
\text { > WIN 55, 212-2 } \\
>\Delta^{9} \text {-THC }\end{array}$ & [41] \\
\hline СР47,497 & $2.5 \mathrm{mg} / \mathrm{kg}$ & Rat & Acute & Locomotion and catalepsy & $\begin{array}{l}>\text { JWH-018 } \\
>\Delta^{9}-\mathrm{THC}\end{array}$ & [42] \\
\hline Cannabicyclo-hexanol & $2.5 \mathrm{mg} / \mathrm{kg}$ & Rat & Acute & Locomotion and catalepsy & $\begin{array}{l}>\mathrm{CP} 47,497 \\
>\text { JWH-018 } \\
>\Delta^{9}-\mathrm{THC}\end{array}$ & [42] \\
\hline \multirow{2}{*}{ JWH-073 } & 3.2-32 mg/kg (i.v.) & Monkey & Acute & Drug discrimination & $>\Delta^{9}-\mathrm{THC}$ & [40] \\
\hline & $0.1-5 \mathrm{mg} / \mathrm{kg}$ & Rat & Acute & $\begin{array}{l}\text { Locomotor activity, Anxiety } \\
\text { and Sensorimotor gating }\end{array}$ & $\begin{array}{l}\text { Reduced locomotor } \\
\text { activity }\end{array}$ & [51] \\
\hline JWH-210 & $0.1-5 \mathrm{mg} / \mathrm{kg}$ & Rat & Acute & $\begin{array}{l}\text { Locomotor activity, Anxiety } \\
\text { and Sensorimotor gating }\end{array}$ & $\begin{array}{l}\text { Reduced locomotor } \\
\text { activity }\end{array}$ & [51] \\
\hline AB-001 & $0.3-30 \mathrm{mg} / \mathrm{kg}$ (i.p.) & Mouse & Acute & Hypothermia & $\begin{array}{l}>\text { JWH-018 } \\
>\Delta^{9}-\mathrm{THC}\end{array}$ & [52] \\
\hline JWH-081 & $0.625-1.25 \mathrm{mg} / \mathrm{kg}$ (i.p.) & mouse & Acute & LTP, Learning and memory & Impaired & [53] \\
\hline AM-4054 & $0.1-1 \mathrm{mg} / \mathrm{kg}$ (s.c.) & Mouse & Chronic & Analgesia & Induced antinociception & [54] \\
\hline AM-4054 & $0.01-0.16 \mathrm{mg} / \mathrm{kg}$ (i.p.) & Rat & Acute & Two-choice operant & Impaired & [55] \\
\hline AM-7418 & $0.03-1 \mathrm{mg} / \mathrm{kg}$ (s.c.) & Mouse & Chronic & Analgesia & Induced antinociception & [54] \\
\hline AM-411 & $0.32-1 \mathrm{mg} / \mathrm{kg}$ (i.m.) & Monkey & $\begin{array}{l}\text { Acute and } \\
\text { Chronic }\end{array}$ & Drug tolerance & $\begin{array}{c}>\text { WIN } 55,212-2 \\
>\Delta^{9}-\text { THC }\end{array}$ & [56] \\
\hline AM-4054 & $0.0032-0.1 \mathrm{mg} / \mathrm{kg}$ (i.m.) & Monkey & $\begin{array}{l}\text { Acute and } \\
\text { Chronic }\end{array}$ & Drug tolerance & $\begin{array}{c}>\text { AM-411 } \\
>\text { WIN } 55,212-2 \\
>\Delta^{9}-\text { THC }\end{array}$ & {$[56]$} \\
\hline AM-2201 & $0.1-1 \mathrm{mg} / \mathrm{kg}$ (s.c.) & Rat & Acute & Hypothermia and Catalepsy & Induced & [57] \\
\hline AM-2201 & $20 \mathrm{nM}-2 \mu \mathrm{M}$ & $\begin{array}{c}\text { Mouse } \\
\text { brain slice }\end{array}$ & Acute & fEPSP & Impaired & [49] \\
\hline UR-144 & $5.6 \mathrm{mg} / \mathrm{kg}$ (i.p.) & Mouse & Acute & $\begin{array}{l}\text { Cannabinoid tetrad and Drug } \\
\text { discrimination }\end{array}$ & $>\Delta^{9}-\mathrm{THC}$ & [58] \\
\hline \multirow[t]{2}{*}{ XLR-11 } & $5.6 \mathrm{mg} / \mathrm{kg}$ (i.p.) & Mouse & Acute & $\begin{array}{l}\text { Cannabinoid tetrad and Drug } \\
\text { discrimination }\end{array}$ & $\begin{array}{l}=\mathrm{UR}-144 \\
>\Delta^{9}-\mathrm{THC}\end{array}$ & [59] \\
\hline & $20 \mathrm{nM}-5 \mu \mathrm{M}$ & Mouse & Acute & fEPSP & Impaired & [49] \\
\hline
\end{tabular}


Table 3. Cont

\begin{tabular}{|c|c|c|c|c|c|c|}
\hline SPCs & Dose & Animal & Exposure & Parameter & Effects & Reference \\
\hline JWH-122 & $0.01-25 \mu \mathrm{M}$ & Human & $\begin{array}{l}\text { Endometrial } \\
\text { stromal cell line }\end{array}$ & Stress and Cell death & $\begin{array}{l}\text { Enhanced stress. No } \\
\text { effect on cell death }\end{array}$ & [60] \\
\hline 5F-MDMB-PINACA & 1.1 mg/kg (i.p.) & Rat & Acute & Locomotion & Reduced (30 min) & [61] \\
\hline MDMB-CHIMICA & $0.024 \mathrm{mg} / \mathrm{kg}$ (i.p.) & Rat & Acute & Locomotion & Reduced (30 min) & [61] \\
\hline ADB-FUBINACA & $0.19 \mathrm{mg} / \mathrm{kg}$ (i.p.) & Rat & Acute & Locomotion & Reduced (60-90 min) & [61] \\
\hline AMB-FUBINACA & $0.19 \mathrm{mg} / \mathrm{kg}$ (i.p.) & Rat & Acute & Locomotion & Reduced (60-90 min) & [61] \\
\hline MDMB-FUBINACA & $0.04 \mathrm{mg} / \mathrm{kg}$ (i.p.) & Rat & Acute & Locomotion & Reduced (150 min) & [61] \\
\hline 5F-AB-PINACA & 10 mg/kg (i.p.) & Mice & Acute & Convulsion & Induced & [46] \\
\hline \multirow{2}{*}{ AB-PINACA } & 1-10 mg/kg (i.p.) & Mice & Acute & Hypothermia & Induced & {$[62]$} \\
\hline & $0.2 \mathrm{mg} / \mathrm{kg}$ & Rat & Chronic & $\begin{array}{l}\text { Learning and memory } \\
\text { Locomotion } \\
\text { Anxiety }\end{array}$ & $\begin{array}{l}\text { Impaired } \\
\text { Decreased } \\
\text { Decreased }\end{array}$ & [63] \\
\hline 4-OH-AB-PINACA & 30 and $10 \mathrm{mg} / \mathrm{kg}$ (i.p.) & Mice & Acute & Hypothermia & induced & [62] \\
\hline 5F-AMB & $300 \mathrm{nM}$ & Mice mPFC slices & Acute & $\begin{array}{l}\text { Excitatory and inhibitory } \\
\text { synaptic transmission }\end{array}$ & $\begin{array}{l}\text { Impaired sEPSP, mEPSP, } \\
\text { sIPSP and mIPSC }\end{array}$ & [64] \\
\hline MMB-FUBINACA & Dose responses (i.p.) & Mice & Acute & Drug discrimination & Substituted for THC & [65] \\
\hline CUMYL-PICA & Dose responses (i.p.) & Mice & Acute & Drug discrimination & Substituted for THC & [65] \\
\hline 5F-CUMYL-PICA & Dose responses (i.p.) & Mice & Acute & Drug discrimination & Substituted for THC & [65] \\
\hline NNEI & Dose responses (i.p.) & Mice & Acute & Drug discrimination & Substituted for THC & [65] \\
\hline MN-18 & Dose responses (i.p.) & Mice & Acute & Drug discrimination & Substituted for THC & [65] \\
\hline AB-FUBINACA & 4.0 mg/kg (i.p.) & Rat & Chronic & $\begin{array}{l}\text { Learning and memory } \\
\text { Locomotion anxiety }\end{array}$ & $\begin{array}{l}\text { Impaired } \\
\text { Decreased } \\
\text { Decreased }\end{array}$ & [66] \\
\hline AB-CHMINACA & 1.0 mg/kg (i.p.) & Rat & Chronic & $\begin{array}{c}\text { Hypothermia } \\
\text { Antinociception } \\
\text { Anxiety } \\
\text { Spatial memory } \\
\text { depression }\end{array}$ & $\begin{array}{l}\text { Induced } \\
\text { No effect } \\
\text { Reduced } \\
\text { Impaired } \\
\text { No effect }\end{array}$ & [66] \\
\hline
\end{tabular}


Table 3. Cont

\begin{tabular}{|c|c|c|c|c|c|c|}
\hline SPCs & Dose & Animal & Exposure & Parameter & Effects & Reference \\
\hline PB-22 & 0.4 mg/kg (i.p.) & Rat & Chronic & $\begin{array}{l}\text { Hypothermia } \\
\text { Antinociception } \\
\text { Anxiety } \\
\text { Spatial memory } \\
\text { Depression }\end{array}$ & $\begin{array}{l}\text { Induced } \\
\text { No effect } \\
\text { Reduced } \\
\text { Impaired } \\
\text { Induced }\end{array}$ & [66] \\
\hline $\begin{array}{l}\text { JWH-018 } \\
\text { JWH-018-R }\end{array}$ & $0.01-1 \mathrm{mg} / \mathrm{kg}$ (i.p.) & Mice & Acute & $\begin{array}{l}\text { Locomotion } \\
\text { Learning and memory } \\
\text { LTP }\end{array}$ & $\begin{array}{l}\text { Impaired } \\
\text { Impaired } \\
\text { Impaired }\end{array}$ & [67] \\
\hline $\begin{array}{l}\text { JWH018 Cl } \\
\text { JWH-018 Br }\end{array}$ & $0.01-1 \mathrm{mg} / \mathrm{kg}$ (i.p.) & Mice & Acute & $\begin{array}{l}\text { Hypothermia } \\
\text { Catalepsy } \\
\text { Locomotion }\end{array}$ & $\begin{array}{l}\text { Induced } \\
\text { Induced } \\
\text { Impaired }\end{array}$ & {$[68]$} \\
\hline JWH-018 & $0.01-6 \mathrm{mg} / \mathrm{kg}$ (i.p.) & Mice & Acute & $\begin{array}{c}\text { Convulsions } \\
\text { Seizures } \\
\text { Hyperreflexia Myoclonias } \\
\text { Visual placing response } \\
\text { Visual object response } \\
\text { Acoustic } \\
\text { Response } \\
\text { Locomotion }\end{array}$ & $\begin{array}{l}\text { Induced } \\
\text { Induced } \\
\text { Induced } \\
\text { Induced } \\
\text { Reduced } \\
\text { Reduced } \\
\text { Reduced } \\
\text { Reduced }\end{array}$ & [69] \\
\hline $\begin{array}{c}\text { AKB48 } \\
\text { 5F-AKB48 }\end{array}$ & $0.01-6 \mathrm{mg} / \mathrm{kg}$ (i.p.) & Mice & Acute & $\begin{array}{c}\text { Convulsions Hyperreflexia } \\
\text { Myoclonias } \\
\text { Catalepsy } \\
\text { Hypothermia } \\
\text { Immobility } \\
\text { Acoustic response } \\
\text { Visual placing response } \\
\text { DA release }\end{array}$ & $\begin{array}{l}\text { Induced } \\
\text { Induced } \\
\text { Induced } \\
\text { Induced } \\
\text { Induced } \\
\text { Induced } \\
\text { Reduced } \\
\text { Reduced } \\
\text { Increased }\end{array}$ & [70] \\
\hline JWH-250 and JWH-073 & 0.01-15 mg/kg (i.p.) & Mice & Acute & $\begin{array}{c}\text { Convulsions Hyperreflexia } \\
\text { Myoclonias Aggressive } \\
\text { responses } \\
\text { Visual object response } \\
\text { Visual placing response } \\
\text { Hypothermia } \\
\text { DA release }\end{array}$ & $\begin{array}{c}\text { Induced } \\
\text { Induced } \\
\text { Induced } \\
\text { Induced } \\
\text { Induce } \\
\text { Induced } \\
\text { Reduced } \\
\text { Increased }\end{array}$ & [71] \\
\hline
\end{tabular}


Table 3. Cont.

\begin{tabular}{|c|c|c|c|c|c|c|}
\hline SPCs & Dose & Animal & Exposure & Parameter & Effects & Reference \\
\hline $\begin{array}{c}\text { JWH018 } \\
\text { AKB48 }\end{array}$ & $0.03-1 \mathrm{mg} / \mathrm{kg}$ (i.p.) & Mice & Acute & $\begin{array}{l}\text { Locomotion } \\
\text { DA release }\end{array}$ & $\begin{array}{l}\text { Increased } \\
\text { Increased }\end{array}$ & [72] \\
\hline $\begin{array}{l}\text { 5F-ADBINACA AB } \\
\text { FUBINACA STS- } 135\end{array}$ & $0.01-6 \mathrm{mg} / \mathrm{kg}$ (i.p.) & Mice & Acute & $\begin{array}{c}\text { Hypothermia } \\
\text { Catalepsy } \\
\text { Locomotion } \\
\text { Sensorimotor responses } \\
\text { Pain threshold } \\
\text { Seizures } \\
\text { Myoclonia Hyperreflexia } \\
\text { Aggressiveness }\end{array}$ & $\begin{array}{l}\text { Induced } \\
\text { Induced } \\
\text { Reduced } \\
\text { Reduced } \\
\text { Increased } \\
\text { Induced } \\
\text { Induced } \\
\text { Induced } \\
\text { Increased }\end{array}$ & [73] \\
\hline
\end{tabular}

potential; i.p.: Intraperitoneal; i.v.: intravenous; s.c.: Subcutaneous; i.m.: Intramuscular. 
JWH-081 produces acute toxicity, as demonstrated by emergency patients, perhaps as a result of strong CB1R activation [34]. JWH-081 binds to CB1R with high affinity $(1.2 \mathrm{nM})[74,75]$. Bath application (acute) of JWH-081 to hippocampal slices impaired long-term potentiation (LTP). This effect was absent in CB1R KO mice slices. Thus, the adverse effects of JWH-081 on LTP are through CB1R activation. Acute JWH-081 administration in adult mice impairs spontaneous alternation as well as spatial memory in the $Y$ maze and object recognition memory. The participation of pCaMKIV, pCREB, and pERK1/2, which are critical for synaptic plasticity, learning, and memory [76] in the acute effects of JWH-081 has also been demonstrated. JWH-081 impaired pCaMKIV and pCREB levels in the hippocampi of CB1R WT but not KO mice. JWH-081 decreased pCaMKIV levels in a dose-dependent manner, while the reduced pCREB was found only at a higher dose of JWH-081 (1.25 mg/kg, $30 \mathrm{~min})$. JWH-081 failed to alter pERK levels and total ERK protein levels in the hippocampi of CB1R WT or KO mice. Further, preadministration of the CB1R antagonist SR141716A 30 min before JWH-081 treatment augmented both loss of pCaMKIV and pCREB levels. JWH-081 exhibited greater in vitro and in vivo responses as compared to $\Delta^{9}$-THC [77]. Overall, the CaMKIV-mediated phosphorylation of CREB at Ser133 is crucial for the transcriptional activation of the CREB/CRE-mediated gene expression [78] and it has been established to play an essential role in memory consolidation and LTP [76]. $\Delta^{9}$-THC significantly reduced the pCREB levels [79] and another calmodulin kinase-related molecules, such as pCaMKII, in a CB1R-dependent manner [80]. All $\Delta^{9}$-THC metabolites, except one, are inactivated through oxidative metabolism [81], which block further CB1R stimulation. The higher affinity, potency, and efficacy of JWH-081 [82], coupled with its likelihood to be metabolized into other metabolites [83], demonstrates that both the acute and chronic effects of JWH-081 might be more important than those of a similar dose of $\Delta^{9}$-THC. Hence, JWH-081 impairs pCaMKIV and pCREB activities through a signaling mechanism downstream of CB1R to elicit potent deleterious neurobehavioral effects in mice (Figure 6). The SCBs, JWH-018, AM2201, and XLR-11, all inhibit glutamate release and impair LTP in the mouse hippocampus in a CB1R-dependent manner [49]. JWH-018 and its halogenated derivatives (JWH-018-Cl, JWH-018-Br) dose-dependently impaired both short- and long-memory retention in mice and diminished electrically evoked synaptic transmission, LTP, glutamate, and GABA release in hippocampal slices [67].

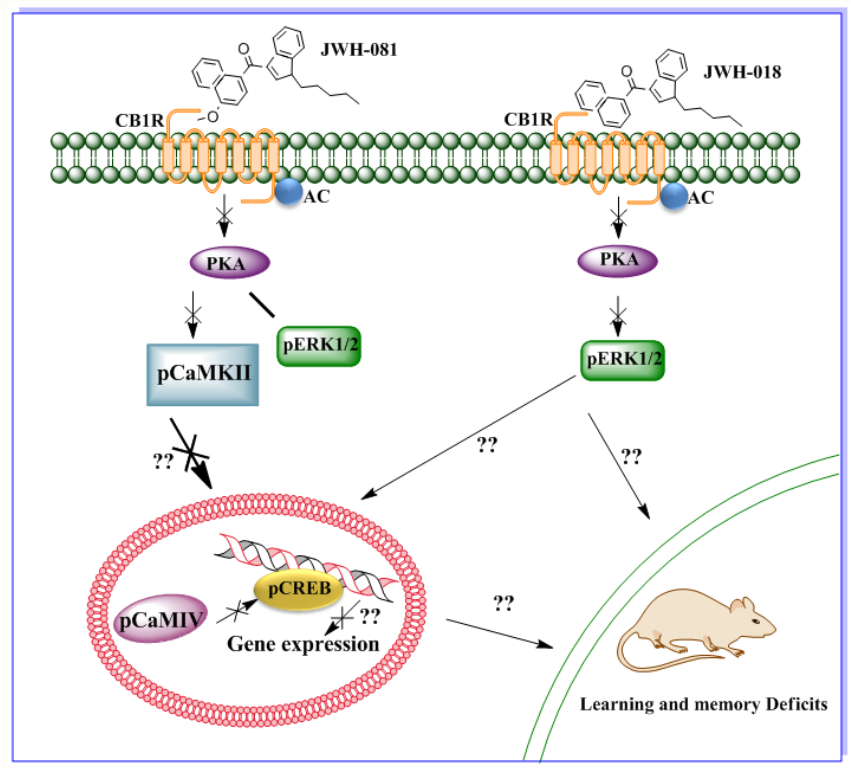

Figure 6. Schematic diagram showing the CB1R signaling mechanism of the SCBs found in Spice products. JWH-081 and JWH-018 both act on CB1R but activate distinct CB1R signaling events. JWH-018, which has a higher affinity for CB2R than for CB1R, reduces pERK1/2. JWH-081, which has a stronger affinity for CB1R than for CB2R, does not affect pERK1/2 but does impair pCaMKIV and pCREB levels, which are linked with Arc gene expression. 
Another derivative of JWH, JWH-018, displays agonistic activity at CB1R (9 nM) and CB2R $(2.94 \mathrm{nM})[84]$ and it produces the tetrad of behaviors that are classically associated with CBs in rodent models (analgesia, catalepsy, hypomotility, and hypothermia) [39,43], but these effects are less potent than those that are associated with JWH-081. Chronic JWH-018 treatment also induces deficits in spatial memory in adolescent mice [85]. JWH-018 inhibits forskolin-stimulated cAMP production [86] and has been shown to reduce pERK levels in cultured hippocampal neurons [48]. While the effects of JWH-081 and JWH-018 are evidently due to CB1R activation, the likely function of CB2R in the effects of compounds that are present in "Spice" and "K2" warrant future investigation.

Another SCB, MAM-2201 (1-(5-fluoropentyl)-1H-indol-3-yl)(4-methyl-1-naphthalenyl)-methanone, suppressed neurotransmitter release at CB1R-expressing Purkinje cell (PC) synapses in cerebellum slices. MAM-2201 caused more significant inhibition of neurotransmitter release than $\triangle 9$-THC and JWH-018. The reduced neurotransmitter release from CB1R-containing PC synapses could contribute to some of the symptoms of SCB intoxication, including impairments in cerebellum-dependent motor coordination and motor learning [87]. Several SCBs have been shown to be cytotoxic in in vitro studies. SCBs, such as CP-47,497 and CP-47,497-C8, exhibited caspase-3- and CB1R-dependent cytotoxicity in NG 108-15 cells, suggesting that caspase cascades may have a significant function in the apoptosis induced by these SCBs [88]. XLR-11, at biologically appropriate doses (in the nanomolar range), has been shown to affect mitochondrial function in human proximal tubule (HK-2) cells through a CBR- and eCB-related mechanism. XLR-11 has been shown to induce a transient hyperpolarization of the mitochondrial membrane and enhance ATP production, followed by Bax translocation from the cytosol into the mitochondria. These events cause energy-dependent apoptotic cell death via mechanisms, such as increased caspase-3 activity and chromatin condensation [89]. Furthermore, JWH-018, JWH-073, and several major human metabolites of these compounds exhibited a high affinity for CB2Rs in CHO-hCB2 cells, suggesting that, when CB2R is available in abundance, these SCBs and their metabolites readily bind to CB2R with high affinity and elicit CB2R signaling [90]. The SCB AM2201 has been shown to induce epileptic seizures by enhancing CB1R-mediated glutamatergic transmission in the hippocampus [91].

The SCB CP-47,497-C8 was shown to induce chromosomal damage, suggesting that SCB could cause genetic instability in SCB abusers [92]. Halogenated derivatives of JWH-018 (JWH-018 Cl and JWH-018 Br) have been shown to impair motor activity and induce catalepsy in mice, and their effects were more severe than those of $\Delta^{9}$-THC. When compared to JWH-018, JWH-018Br was less effective at causing seizures, myoclonia, and hyperreflexia. These findings suggest that the halogenated compounds might have been used in the Spice products to produce similar intoxicating effects as JWH-018 while producing fewer side effects [68]. In another in vivo study, repeated administration of JWH-018 transiently enhanced 5-HT1A receptor sensitivity to produce tolerance to its hypothermic and cataleptic effects [36]. It has been shown that JWH-018 undergoes extensive metabolism by cytochrome P450 (P450). The major enzyme involved in this metabolism is CYP2C9, a highly polymorphic enzyme that is found largely in the intestines and liver. The polymorphic nature of CYP2C9 results in variable levels of biologically active JWH-018 metabolites in some individuals, offering a mechanistic explanation for the diverse clinical toxicity often observed following JWH-018 abuse [93]. Recently developed SCBs, such as MMB-FUBINACA, MDMB-FUBINACA, CUMYL-PICA, 5F-CUMYL-PICA, NNEI, and MN-18, exhibited a high affinity for human CB1R and CB2R and produced greater effects than $\Delta^{9}$-THC in $\left.{ }^{35} \mathrm{~S}\right] \mathrm{GTP} \gamma \mathrm{S}$ binding and cAMP signaling assays. Additionally, all six synthetic cannabinoids replaced $\Delta^{9}$-THC in drug discrimination, suggesting that these SCBs may possess subjective effects that are comparable to those of cannabis. Notably, MDMB-FUBINACA, a methylated analog of MMB-FUBINACA, exhibited a higher affinity for CB1R than the parent molecule, suggesting that slight structural alterations could cause a larger impact on the pharmacological properties of these drugs [65]. AM2201 and XLR-11 resulted in hypothermia in a CB1R-dependent manner in mice [94]. JWH-018 administration inhibited sensorimotor responses at lower doses, reduced spontaneous locomotion at intermediate/high doses, and induced convulsions, myoclonia and hyperreflexia at 
high doses in male CD-1 mice. These SCB effects were CB1R-dependent and directly implicated in SCB abuse and driving [69]. JWH-250 and JWH-073 exhibited nanomolar affinity and potency in in vitro competition binding experiments that were performed on a mouse and human CB1R and CB2R. In vivo administration of either JWH-250 or JWH-073 induced marked hypothermia and increased the pain threshold to both noxious mechanical and thermal stimuli. In addition, it also caused catalepsy, reduced motor activity, impaired sensorimotor responses (visual, acoustic and tactile), caused seizures, myoclonia, and hyperreflexia; and, promoted aggressiveness in male CD-1 mice. Furthermore, a microdialysis study in freely moving mice showed that systemic administration of JWH-250 or JWH-073 stimulated dopamine release in the nucleus accumbens. The CB1R agonist AM251 fully rescued behavioral, neurological, and neurochemical effects. Furthermore, coadministration of ineffective doses of JWH-250 and JWH-073 impaired visual sensory motor responses, augmented the mechanical pain threshold, stimulated mesolimbic DA transmission, and affected other behavioral and physiological parameters [71]. In other in vivo studies, JWH-018 and AKB48 facilitated spontaneous locomotion in mice. These behavioral effects were inhibited by CB1R and dopamine (DA) $\mathrm{D}_{1} / 5$ and $\mathrm{D}_{2} / 3$ receptor blockade. SPECT-CT studies with dopamine transporter (DAT) revealed that JWH-018 and AKB48 decreased [ $\left.{ }^{123} \mathrm{I}\right]-\mathrm{FP}-\mathrm{CIT}$ binding in the mouse striatum. Moreover, microdialysis studies showed that the in vivo administration of JWH-018 or AKB48 activated DA release in the nucleus accumbens (NAc) of freely moving mice. These results suggest that JWH-018 and AKB48 induced a psychostimulant effect in mice, possibly by increasing NAc DA release [72]. In other in vivo studies, 5F-ADBINACA, AB-FUBINACA, and STS-135 caused hypothermia; enhanced pain threshold to both noxious mechanical and thermal stimuli; induced catalepsy; impaired motor activity; resulted in deficits in sensorimotor responses (visual, acoustic, and tactile); induced seizures, myoclonia, and hyperreflexia; and, promoted aggressiveness through CB1R in mice [70]. However, the visual sensory response that is induced by STS-135 was only partially prevented by AM 251, suggesting an additional CB1R-independent mechanism [73]. Two recently developed SCBs (AKB48 and 5F-AKB48) exhibited nanomolar affinity and potency at CBRs in mouse and human cell preparations in in vitro competition binding experiments. Administration of AKB48 or 5F-AKB48 in CD-1 mice elicited hypothermia; enhanced the pain threshold to both noxious mechanical and thermal stimuli; induced catalepsy; impaired motor activity; resulted in defects in sensorimotor responses (visual, acoustic and tactile); elicited seizures, myoclonia, and hyperreflexia; and, promoted aggressiveness in mice. Moreover, a microdialysis study in freely moving mice showed that in vivo administration of AKB48 and 5F-AKB48 increased dopamine release in the nucleus accumbens. These behavioral, neurological, and neurochemical effects were inhibited by the administration of a CB1R antagonist (AM 251). Interestingly, a new generation of novel carboxamide synthetic cannabinoids have continued [61] to appear in the market as marijuana alternatives to evade drug control laws and cannabinoid blood/urine tests. For example, 5F-MDMB-PINACA (also known as 5F-ADB, 5F-ADB-PINACA), MDMB-CHIMICA, MDMB-FUBINACA, ADB-FUBINACA, and AMB-FUBINACA (also known as FUB-AMB and MMB-FUBINACA) were found to exhibit in vivo cannabinoid-like effects. Although all of these SCBs depressed locomotor activities, the time that is required for them to induce depressive activity varies. For example, 5F-MDMB-PINACA and MDMB-CHIMICA induced (30 min) depression of locomotor activity. ADB-FUBINACA and AMB-FUBINACA depressed locomotor activity for 60-90 min, whereas MDMB-FUBINACA depressed locomotor activity for 150 min. Furthermore, AMB-FUBINACA induced tremors at the highest dose tested. Furthermore, 5F-MDMB-PINACA, MDMB-CHIMICA, MDMB-FUBINACA, ADB-FUBINACA, and AMB-FUBINACA completely replaced the discriminative stimulus effects of $\Delta^{9}$-THC following 15-min preadministration. Together, these findings suggest that these SCBs may have abuse potential that is comparable to that of $\Delta^{9}$-THC. The AMB-FUBINACA may induce an increased risk of harmfulness in recreational users [61].

Abused SCBs have been shown to cause convulsion, but the mechanisms are not well understood. A recent study compared the convulsant effects of $\Delta^{9}-\mathrm{THC}, \mathrm{JWH}-018$, and 5F-AB-PINACA as well as that of a classic chemical convulsant pentylenetetrazol (PTZ) using an observational rating scale 
in mice [46]. JWH-018 and 5F-AB-PINACA elicited severe convulsions when compared to those elicited by PTZ, and $\Delta^{9}$-THC failed to elicit convulsions. SR141716A, but not PTZ, blocked the effects of JWH-018 and 5F-AB-PINACA. Nonselective inhibition of CYP450s with 1-aminobenzotriazole (1-ABT) potentiated the hypothermic effects of JWH-018 and 5F-AB-PINACA and provided protection against the convulsant effects of JWH-018 but not those of 5F-AB-PINACA or PTZ. These findings suggest that SCB-elicited convulsions are facilitated by high intrinsic efficacy at CB1Rs and indicate that drug metabolism plays an essential role in the in vivo efficacy of SCBs. Systemic administration of JWH-073, JWH-210, and $\Delta^{9}$-THC failed to affect sensorimotor gating, and locomotor activity was only partially affected. However, $\Delta^{9}-\mathrm{THC}$, but not JWH-073 and JWH-210, induced an anxiolytic-like effect [51]. In vitro experiments have suggested that AB-PINACA displays a comparable affinity for CB1Rs but a greater efficacy for G-protein activation and a higher potency for adenylyl cyclase (AC) inhibition than $\Delta^{9}$-THC. Chronic administration of AB-PINACA also causes greater desensitization of CB1Rs (e.g., tolerance) than chronic administration of $\Delta^{9}$-THC. Notably, monohydroxy metabolites of AB-PINACA retain their affinity and full agonist activity at CB1Rs. Furthermore, the systemic administration of AB-PINACA and 4OH-AB-PINACA caused hypothermia in a CB1R-dependent manner [62]. These data demonstrate that AB-PINACA exhibits pronounced adverse effects through atypical pharmacodynamic properties at CB1Rs as compared to the effects of $\Delta^{9}$-THC by producing metabolically stable active phase I metabolites. These findings indicate that SCBs found in Spice products may exert their acute deleterious effect through CBR. However, a limited number of long-term studies have suggested that SCBs may induce severe harmful effects via complex neuroadaptation mechanisms, and future investigations on this topic are necessary.

In summary, investigation on the psychoactive components of marijuana, as well as the role of the $\mathrm{eCB}$ system in humans and its relationship to various brain disorders, has received much attention since the identification of $\mathrm{CB}$ receptors and their eCB ligands. Moreover, in addition to the well-known symptoms of euphoria and pleasure, SCBs binding to CB1R can also cause anxiety, short-term memory loss, and attention deficits and can have many other cognitive, affective, and psychomotor effects. The extent to which brain development and functions are disturbed remains unknown in young SCB users who have been shown to develop an increased risk of $C B$ dependence. Although studies on the acute effects of SCBs are underway, studies on the chronic abuse of SCB drugs are still insufficient; thus, this area requires future attention. The immediate results of acute SCBs are probably related to the stimulation of presynaptic CB1R-mediated signaling cascades and the inhibition of the release of several neurotransmitters in the brain. In chronic SCB users, it can be presumed that adaptive changes in the CB1R-mediated signaling cascades and the related neurotransmitter systems result in severe adverse effects. It is likely that the additional compounds that were identified in the Spice/K2 preparations might also contribute, through CB1R or CB2R signaling, to the behavioral effects that are produced by smoking "Spice/K2". In addition, their different pharmacological characteristics might cause the harmful effects of different preparations of "Spice/K2" to vary. Further investigation into these additional SCBs is required. "Spice/K2" is marketed as a "natural" herbal blend but contains at least one very potent SCB that acts through CB1R signaling, which likely accounts for the severe deleterious effects that are elicited by smoking "Spice/K2".

Author Contributions: Conceptualization, Writing-Review (B.S.B.) and Editing (B.S.B. and S.S.).

Funding: This work was supported in part by NIH/NIAAA grant AA019443 (B.S.B.).

Conflicts of Interest: The authors declare no conflict of interest.

\section{References}

1. Johnston, L.D.; O’Malley, P.M.; Bachman, J.G.; Schulenberg, J.E. Marijuana Use Continues to Rise Among U.S. Teens, While Alcohol Use Hits Historic Lows; University of Michigan News Service: Ann Arbor, MI, USA, 2012; Available online: https://news.umich.edu/marijuana-use-continues-to-rise-among-us-teens-while-alcoholuse-hits-historic-lows/ (accessed on 5 October 2012). 
2. Gaoni, Y.; Mechoulam, R. Isolation, structure and partial synthesis of an active constituent of hashish. J. Am. Chem. Soc. 1964, 86, 1646-1647. [CrossRef]

3. Mehmedic, Z.; Chandra, S.; Slade, D.; Denham, H.; Foster, S.; Patel, A.S.; Ross, S.A.; Khan, I.A.; ElSohly, M.A. Potency trends of delta9-THC and other cannabinoids in confiscated cannabis preparations from 1993 to 2008. J. Forensic. Sci. 2010, 55, 1209-1217. [CrossRef]

4. Pertwee, R.G. The central neuropharmacology of psychotropic cannabinoids. Pharmacol. Ther. 1988, 36, 189-261. [CrossRef]

5. Basavarajappa, B.S. Major enzymes of endocannabinoid metabolism. In Frontiers in Protein and Peptide Sciences; Dunn, B., Ed.; Bentham Science Publishers: Oak Park, IL, USA, 2014; Volume 1, pp. 31-62.

6. Herkenham, M.; Lynn, A.B.; de Costa, B.R.; Richfield, E.K. Neuronal localization of cannabinoid receptors in the basal ganglia of the rat. Brain Res. 1991, 547, 267-274. [CrossRef]

7. Basavarajappa, B.S. Neuropharmacology of the endocannabinoid signaling system-molecular mechanisms, biological actions and synaptic plasticity. Curr. Neuropharmacol. 2007, 5, 81-97. [CrossRef]

8. Battistella, G.; Fornari, E.; Annoni, J.M.; Chtioui, H.; Dao, K.; Fabritius, M.; Favrat, B.; Mall, J.F.; Maeder, P.; Giroud, C. Long-term effects of cannabis on brain structure. Neuropsychopharmacology 2014, 39, 2041-2048. [CrossRef]

9. Herkenham, M.; Lynn, A.B.; Johnson, M.R.; Melvin, L.S.; de Cost, B.R.; Rice, K.C. Characterization and localization of cannabinoid receptors in rat brain: A quantitative in vitro autoradiographic study. J. Neurosci. 1991, 16, 8057-8066. [CrossRef]

10. Onaivi, E.S.; Ishiguro, H.; Gong, J.P.; Patel, S.; Meozzi, P.A.; Myers, L.; Perchuk, A.; Mora, Z.; Tagliaferro, P.A.; Gardner, E.; et al. Brain neuronal CB2 cannabinoid receptors in drug abuse and depression: From mice to human subjects. PLoS ONE 2008, 3, e1640. [CrossRef]

11. Ramirez, S.H.; Hasko, J.; Skuba, A.; Fan, S.; Dykstra, H.; McCormick, R.; Reichenbach, N.; Krizbai, I.; Mahadevan, A.; Zhang, M.; et al. Activation of cannabinoid receptor 2 attenuates leukocyte-endothelial cell interactions and blood-brain barrier dysfunction under inflammatory conditions. J. Neurosci. 2012, 32, 4004-4016. [CrossRef]

12. Li, Y.; Kim, J. Neuronal expression of CB2 cannabinoid receptor mrnas in the mouse hippocampus. Neuroscience 2015, 311, 253-267. [CrossRef]

13. Zhang, H.Y.; Gao, M.; Liu, Q.R.; Bi, G.H.; Li, X.; Yang, H.J.; Gardner, E.L.; Wu, J.; Xi, Z.X. Cannabinoid CB2 receptors modulate midbrain dopamine neuronal activity and dopamine-related behavior in mice. Proc. Natl. Acad. Sci. USA 2014, 111, E5007-E5015. [CrossRef]

14. Zhang, H.Y.; Gao, M.; Shen, H.; Bi, G.H.; Yang, H.J.; Liu, Q.R.; Wu, J.; Gardner, E.L.; Bonci, A.; Xi, Z.X. Expression of functional cannabinoid $\mathrm{CB}_{2}$ receptor in VTA dopamine neurons in rats. Addict. Biol. 2016, 22, 752-756. [CrossRef]

15. Li, Y.; Kim, J. CB2 cannabinoid receptor knockout in mice impairs contextual long-term memory and enhances spatial working memory. Neural Plast. 2016, 2016, 1-14. [CrossRef]

16. Li, Y.; Kim, J. Deletion of CB2 cannabinoid receptors reduces synaptic transmission and long-term potentiation in the mouse hippocampus. Hippocampus 2016, 26, 275-281. [CrossRef]

17. Stempel, A.V.; Stumpf, A.; Zhang, H.Y.; Ozdogan, T.; Pannasch, U.; Theis, A.K.; Otte, D.M.; Wojtalla, A.; Racz, I.; Ponomarenko, A.; et al. Cannabinoid type 2 receptors mediate a cell type-specific plasticity in the hippocampus. Neuron 2016, 90, 795-809. [CrossRef]

18. Van Sickle, M.D.; Duncan, M.; Kingsley, P.J.; Mouihate, A.; Urbani, P.; Mackie, K.; Stella, N.; Makriyannis, A.; Piomelli, D.; Davison, J.S.; et al. Identification and functional characterization of brainstem cannabinoid CB2 receptors. Science 2005, 310, 329-332. [CrossRef]

19. Viscomi, M.T.; Oddi, S.; Latini, L.; Pasquariello, N.; Florenzano, F.; Bernardi, G.; Molinari, M.; Maccarrone, M. Selective CB2 receptor agonism protects central neurons from remote axotomy-induced apoptosis through the PI3K/AKT pathway. J. Neurosci. 2009, 29, 4564-4570. [CrossRef]

20. Kearn, C.S.; Greenberg, M.J.; DiCamelli, R.; Kurzawa, K.; Hillard, C.J. Relationships between ligand affinities for the cerebellar cannabinoid receptor CB1 and the induction of GDP/GTP exchange. J. Neurochem. 1999, 72, 2379-2387. [CrossRef]

21. Mukhopadhyay, S.; McIntosh, H.H.; Houston, D.B.; Howlett, A.C. The CB(1) cannabinoid receptor juxtamembrane C-terminal peptide confers activation to specific G proteins in brain. Mol. Pharmacol. $2000,57,162-170$. 
22. Glass, M.; Felder, C.C. Concurrent stimulation of cannabinoid CB1 and dopamine D2 receptors augments camp accumulation in striatal neurons: Evidence for a Gs linkage to the CB1 receptor. J. Neurosci. 1997, 17, 5327-5333. [CrossRef]

23. Rhee, M.H.; Bayewitch, M.; Avidor-Reiss, T.; Levy, R.; Vogel, Z. Cannabinoid receptor activation differentially regulates the various adenylyl cyclase isozymes. J. Neurochem. 1998, 71, 1525-1534. [CrossRef]

24. Herrera, B.; Carracedo, A.; Diez-Zaera, M.; Gomez del Pulgar, T.; Guzman, M.; Velasco, G. The CB2 cannabinoid receptor signals apoptosis via ceramide-dependent activation of the mitochondrial intrinsic pathway. Exp. Cell Res. 2006, 312, 2121-2131. [CrossRef]

25. Shi, J.; Cai, Q.; Zhang, J.; He, X.; Liu, Y.; Zhu, R.; Jin, L. AM1241 alleviates MPTP-induced Parkinson's disease and promotes the regeneration of DA neurons in PD mice. Oncotarget 2017, 8, 67837-67850.

26. Li, L.; Yun, D.; Zhang, Y.; Tao, Y.; Tan, Q.; Qiao, F.; Luo, B.; Liu, Y.; Fan, R.; Xian, J.; et al. A cannabinoid receptor 2 agonist reduces blood-brain barrier damage via induction of MKP-1 after intracerebral hemorrhage in rats. Brain Res. 2018, 1697, 113-123. [CrossRef]

27. Fujii, M.; Sherchan, P.; Soejima, Y.; Hasegawa, Y.; Flores, J.; Doycheva, D.; Zhang, J.H. Cannabinoid receptor type 2 agonist attenuates apoptosis by activation of phosphorylated CREB-Bcl-2 pathway after subarachnoid hemorrhage in rats. Exp. Neurol. 2014, 261, 396-403. [CrossRef]

28. Stumpf, A.; Parthier, D.; Sammons, R.P.; Stempel, A.V.; Breustedt, J.; Rost, B.R.; Schmitz, D. Cannabinoid type 2 receptors mediate a cell type-specific self-inhibition in cortical neurons. Neuropharmacology 2018, 139, 217-225. [CrossRef]

29. Huffman, J.W.; Dong, D.; Martin, B.R.; Compton, D.R. Design, synthesis and pharmacology of cannabimimetic indoles. Bioorg. Med. Chem. Lett. 1994, 4, 563-566. [CrossRef]

30. Vardakou, I.; Pistos, C.; Spiliopoulou, C. Spice drugs as a new trend: Mode of action, identification and legislation. Toxicol. Lett. 2010, 197, 157-162. [CrossRef]

31. US Drug Enforcement Administration. Schedules of controlled substances:Temporary placement of four synthetic cannabinoids into schedule I Final order. Fed. Regist 2014, 79, 7577-7582.

32. Howlett, A.C.; Barth, F.; Bonner, T.I.; Cabral, G.; Casellas, P.; Devane, W.A.; Felder, C.C.; Herkenham, M.; Mackie, K.; Martin, B.R.; et al. International union of pharmacology. XXVII. Classification of cannabinoid receptors. Pharmacol. Rev. 2002, 54, 161-202. [CrossRef]

33. Castaneto, M.S.; Gorelick, D.A.; Desrosiers, N.A.; Hartman, R.L.; Pirard, S.; Huestis, M.A. Synthetic cannabinoids: Epidemiology, pharmacodynamics, and clinical implications. Drug Alcohol Depend. 2014, 144, 12-41. [CrossRef]

34. Hermanns-Clausen, M.; Kneisel, S.; Szabo, B.; Auwarter, V. Acute toxicity due to the confirmed consumption of synthetic cannabinoids: Clinical and laboratory findings. Addiction 2013, 108, 534-544. [CrossRef]

35. Pertwee, R.G. Receptors and channels targeted by synthetic cannabinoid receptor agonists and antagonists. Curr. Med. Chem. 2010, 17, 1360-1381. [CrossRef]

36. Elmore, J.S.; Baumann, M.H. Repeated exposure to the "spice" cannabinoid JWH-018 induces tolerance and enhances responsiveness to 5-HT1A receptor stimulation in male rats. Front. Psychiatry 2018, 9, 55. [CrossRef]

37. Lopez-Dyck, E.; Andrade-Urzua, F.; Elizalde, A.; Ferrer-Villada, T.; Dagnino-Acosta, A.; Huerta, M.; Osuna-Calleros, Z.; Rangel-Sandoval, C.; Sanchez-Pastor, E. Acpa and JWH-133 modulate the vascular tone of superior mesenteric arteries through cannabinoid receptors, bkca channels, and nitric oxide dependent mechanisms. Pharmacol. Rep. 2017, 69, 1131-1139. [CrossRef]

38. Yun, J.; Yoon, K.S.; Lee, T.H.; Lee, H.; Gu, S.M.; Song, Y.J.; Cha, H.J.; Han, K.M.; Seo, H.; Shin, J.; et al. Synthetic cannabinoid, JWH-030, induces QT prolongation through herg channel inhibition. Toxicol. Res. (Camb.) 2016, 5, 1663-1671. [CrossRef]

39. Brents, L.K.; Reichard, E.E.; Zimmerman, S.M.; Moran, J.H.; Fantegrossi, W.E.; Prather, P.L. Phase i hydroxylated metabolites of the k2 synthetic cannabinoid JWH-018 retain in vitro and in vivo cannabinoid 1 receptor affinity and activity. PLoS ONE 2011, 6, e21917. [CrossRef]

40. Ginsburg, B.C.; Schulze, D.R.; Hruba, L.; McMahon, L.R. Jwh-018 and jwh-073: Delta(9)-tetrahydrocannabinollike discriminative stimulus effects in monkeys. J. Pharmacol. Exp. Ther. 2012, 340, 37-45. [CrossRef]

41. Jarbe, T.U.; Deng, H.; Vadivel, S.K.; Makriyannis, A. Cannabinergic aminoalkylindoles, including AM678 $=\mathrm{JWH} 018$ found in 'spice', examined using drug (delta(9)-tetrahydrocannabinol) discrimination for rats. Behav. Pharmacol. 2011, 22, 498-507. [CrossRef] 
42. Uchiyama, N.; Kikura-Hanajiri, R.; Matsumoto, N.; Huang, Z.; Goda, Y.; Urade, Y. Effects of synthetic cannabinoids, cannabicyclohexanol and jwh-018, on electroencephalogram power spectra and locomotor activity in rats. Sleep Biol. Rhythms. 2011, 9, 342.

43. Wiebelhaus, J.M.; Poklis, J.L.; Poklis, A.; Vann, R.E.; Lichtman, A.H.; Wise, L.E. Inhalation exposure to smoke from synthetic "marijuana" produces potent cannabimimetic effects in mice. Drug Alcohol Depend. 2012, 126, 316-323. [CrossRef]

44. Wiley, J.L.; Marusich, J.A.; Martin, B.R.; Huffman, J.W. 1-pentyl-3-phenylacetylindoles and jwh-018 share in vivo cannabinoid profiles in mice. Drug Alcohol Depend. 2012, 123, 148-153. [CrossRef]

45. Macri, S.; Lanuzza, L.; Merola, G.; Ceci, C.; Gentili, S.; Valli, A.; Macchia, T.; Laviola, G. Behavioral responses to acute and sub-chronic administration of the synthetic cannabinoid jwh-018 in adult mice prenatally exposed to corticosterone. Neurotox Res. 2013, 24, 15-28. [CrossRef]

46. Wilson, C.D.; Tai, S.; Ewing, L.; Crane, J.; Lockhart, T.; Yarbrough, A.L.; Fujiwara, R.; Radominska-Pandya, A.; Fantegrossi, W.E. Convulsant effects of abused synthetic cannabinoids jwh- 018 and $5 \mathrm{f}$-ab-pinaca are mediated by agonist actions at cb1 receptors in mice. J. Pharmacol. Exp. Ther. 2019, 368, 146-156. [CrossRef]

47. Theunissen, E.L.; Hutten, N.; Mason, N.L.; Toennes, S.W.; Kuypers, K.P.C.; de Sousa Fernandes Perna, E.B.; Ramaekers, J.G. Neurocognition and subjective experience following acute doses of the synthetic cannabinoid jwh-018: A phase 1, placebo-controlled, pilot study. Br. J. Pharmacol. 2018, 175, 18-28. [CrossRef]

48. Atwood, B.K.; Huffman, J.; Straiker, A.; Mackie, K. Jwh018, a common constituent of 'spice' herbal blends, is a potent and efficacious cannabinoid cb receptor agonist. Br. J. Pharmacol. 2010, 160, 585-593. [CrossRef]

49. Hoffman, A.F.; Lycas, M.D.; Kaczmarzyk, J.R.; Spivak, C.E.; Baumann, M.H.; Lupica, C.R. Disruption of hippocampal synaptic transmission and long-term potentiation by psychoactive synthetic cannabinoid 'spice' compounds: Comparison with delta(9) -tetrahydrocannabinol. Addict. Biol. 2017, 22, 390-399. [CrossRef]

50. Jarbe, T.U.; Tai, S.; LeMay, B.J.; Nikas, S.P.; Shukla, V.G.; Zvonok, A.; Makriyannis, A. Am2389, a high-affinity, in vivo potent cb1-receptor-selective cannabinergic ligand as evidenced by drug discrimination in rats and hypothermia testing in mice. Psychopharmacology 2012, 220, 417-426. [CrossRef]

51. Uttl, L.; Szczurowska, E.; Hajkova, K.; Horsley, R.R.; Stefkova, K.; Hlozek, T.; Sichova, K.; Balikova, M.; Kuchar, M.; Micale, V.; et al. Behavioral and pharmacokinetic profile of indole-derived synthetic cannabinoids jwh-073 and jwh-210 as compared to the phytocannabinoid delta(9)-thc in rats. Front. Neurosci. 2018, 12, 703. [CrossRef]

52. Banister, S.D.; Wilkinson, S.M.; Longworth, M.; Stuart, J.; Apetz, N.; English, K.; Brooker, L.; Goebel, C.; Hibbs, D.E.; Glass, M.; et al. The synthesis and pharmacological evaluation of adamantane-derived indoles: Cannabimimetic drugs of abuse. ACS Chem. Neurosci. 2013, 4, 1081-1092. [CrossRef]

53. Basavarajappa, B.S.; Subbanna, S. Cb1 receptor-mediated signaling underlies the hippocampal synaptic, learning, and memory deficits following treatment with jwh-081, a new component of spice/k2 preparations. Hippocampus 2014, 24, 178-188. [CrossRef]

54. Chopda, G.R.; Vemuri, V.K.; Sharma, R.; Thakur, G.A.; Makriyannis, A.; Paronis, C.A. Diuretic effects of cannabinoid agonists in mice. Eur. J. Pharmacol. 2013, 721, 64-69. [CrossRef]

55. Miller, R.L.; Thakur, G.A.; Stewart, W.N.; Bow, J.P.; Bajaj, S.; Makriyannis, A.; McLaughlin, P.J. Effects of a novel cb1 agonist on visual attention in male rats: Role of strategy and expectancy in task accuracy. Exp. Clin. Psychopharmacol. 2013, 21, 416-425. [CrossRef] [PubMed]

56. Desai, R.I.; Thakur, G.A.; Vemuri, V.K.; Bajaj, S.; Makriyannis, A.; Bergman, J. Analysis of tolerance and behavioral/physical dependence during chronic cb1 agonist treatment: Effects of cb1 agonists, antagonists, and noncannabinoid drugs. J. Pharmacol. Exp. Ther. 2013, 344, 319-328. [CrossRef] [PubMed]

57. Carlier, J.; Wohlfarth, A.; Salmeron, B.D.; Scheidweiler, K.B.; Huestis, M.A.; Baumann, M.H. Pharmacodynamic effects, pharmacokinetics, and metabolism of the synthetic cannabinoid am-2201 in male rats. J. Pharmacol. Exp. Ther. 2018, 367, 543-550. [CrossRef]

58. Gatch, M.B.; Forster, M.J. Delta9-tetrahydrocannabinol-like effects of novel synthetic cannabinoids found on the gray market. Behav. Pharmacol. 2015, 26, 460-468. [CrossRef]

59. Wiley, J.L.; Marusich, J.A.; Lefever, T.W.; Grabenauer, M.; Moore, K.N.; Thomas, B.F. Cannabinoids in disguise: Delta9-tetrahydrocannabinol-like effects of tetramethylcyclopropyl ketone indoles. Neuropharmacology 2013, 75, 145-154. [CrossRef] 
60. Fonseca, B.M.; Fernandes, R.; Almada, M.; Santos, M.; Carvalho, F.; Teixeira, N.A.; Correia-da-Silva, G. Synthetic cannabinoids and endometrial stromal cell fate: Dissimilar effects of jwh-122, ur-144 and win55,212-2. Toxicology 2018, 413, 40-47. [CrossRef]

61. Gatch, M.B.; Forster, M.J. Cannabinoid-like effects of five novel carboxamide synthetic cannabinoids. Neurotoxicology 2018, 70, 72-79. [CrossRef]

62. Hutchison, R.D.; Ford, B.M.; Franks, L.N.; Wilson, C.D.; Yarbrough, A.L.; Fujiwara, R.; Su, M.K.; Fernandez, D.; James, L.P.; Moran, J.H.; et al. Atypical pharmacodynamic properties and metabolic profile of the abused synthetic cannabinoid ab-pinaca: Potential contribution to pronounced adverse effects relative to delta(9)-thc. Front. Pharmacol. 2018, 9, 1084. [CrossRef]

63. Kevin, R.C.; Wood, K.E.; Stuart, J.; Mitchell, A.J.; Moir, M.; Banister, S.D.; Kassiou, M.; McGregor, I.S. Acute and residual effects in adolescent rats resulting from exposure to the novel synthetic cannabinoids ab-pinaca and ab-fubinaca. J. Psychopharmacol. 2017, 31, 757-769. [CrossRef]

64. Domoto, M.; Sasase, H.; Wada, S.; Ito, S.; Deyama, S.; Hinoi, E.; Kaneko, S.; Kaneda, K. The synthetic cannabinoid $5 \mathrm{f}$-amb changes the balance between excitation and inhibition of layer $\mathrm{V}$ pyramidal neurons in the mouse medial prefrontal cortex. Psychopharmacology 2018, 235, 2367-2376. [CrossRef]

65. Gamage, T.F.; Farquhar, C.E.; Lefever, T.W.; Marusich, J.A.; Kevin, R.C.; McGregor, I.S.; Wiley, J.L.; Thomas, B.F. Molecular and behavioral pharmacological characterization of abused synthetic cannabinoids mmb- and mdmb-fubinaca, mn-18, nnei, cumyl-pica, and 5-fluoro-cumyl-pica. J. Pharmacol. Exp. Ther. 2018, 365, 437-446. [CrossRef]

66. Schreiber, S.; Bader, M.; Lenchinski, T.; Meningher, I.; Rubovitch, V.; Katz, Y.; Cohen, E.; Gabet, Y.; Rotenberg, M.; Wolf, E.U.; et al. Functional effects of synthetic cannabinoids versus delta(9) -thc in mice on body temperature, nociceptive threshold, anxiety, cognition, locomotor/exploratory parameters and depression. Addict. Biol. 2018. [CrossRef]

67. Barbieri, M.; Ossato, A.; Canazza, I.; Trapella, C.; Borelli, A.C.; Beggiato, S.; Rimondo, C.; Serpelloni, G.; Ferraro, L.; Marti, M. Synthetic cannabinoid jwh-018 and its halogenated derivatives jwh-018-cl and jwh-018-br impair novel object recognition in mice: Behavioral, electrophysiological and neurochemical evidence. Neuropharmacology 2016, 109, 254-269. [CrossRef]

68. Vigolo, A.; Ossato, A.; Trapella, C.; Vincenzi, F.; Rimondo, C.; Seri, C.; Varani, K.; Serpelloni, G.; Marti, M. Novel halogenated derivates of jwh-018: Behavioral and binding studies in mice. Neuropharmacology 2015, 95, 68-82. [CrossRef]

69. Ossato, A.; Vigolo, A.; Trapella, C.; Seri, C.; Rimondo, C.; Serpelloni, G.; Marti, M. Jwh-018 impairs sensorimotor functions in mice. Neuroscience 2015, 300, 174-188. [CrossRef]

70. Canazza, I.; Ossato, A.; Trapella, C.; Fantinati, A.; De Luca, M.A.; Margiani, G.; Vincenzi, F.; Rimondo, C.; Di Rosa, F.; Gregori, A.; et al. Effect of the novel synthetic cannabinoids akb48 and 5f-akb48 on "tetrad", sensorimotor, neurological and neurochemical responses in mice. In vitro and in vivo pharmacological studies. Psychopharmacology 2016, 233, 3685-3709. [CrossRef]

71. Ossato, A.; Canazza, I.; Trapella, C.; Vincenzi, F.; De Luca, M.A.; Rimondo, C.; Varani, K.; Borea, P.A.; Serpelloni, G.; Marti, M. Effect of jwh-250, jwh-073 and their interaction on "tetrad", sensorimotor, neurological and neurochemical responses in mice. Prog. Neuropsychopharmacol. Biol. Psychiatry 2016, 67, 31-50. [CrossRef]

72. Ossato, A.; Uccelli, L.; Bilel, S.; Canazza, I.; Di Domenico, G.; Pasquali, M.; Pupillo, G.; De Luca, M.A.; Boschi, A.; Vincenzi, F.; et al. Psychostimulant effect of the synthetic cannabinoid jwh-018 and akb48: Behavioral, neurochemical, and dopamine transporter scan imaging studies in mice. Front. Psychiatry 2017, 8, 130. [CrossRef]

73. Canazza, I.; Ossato, A.; Vincenzi, F.; Gregori, A.; Di Rosa, F.; Nigro, F.; Rimessi, A.; Pinton, P.; Varani, K.; Borea, P.A.; et al. Pharmaco-toxicological effects of the novel third-generation fluorinate synthetic cannabinoids, 5f-adbinaca, ab-fubinaca, and sts-135 in mice. In vitro and in vivo studies. Hum. Psychopharmacol. 2017, 32, e2601. [CrossRef]

74. Aung, M.M.; Griffin, G.; Huffman, J.W.; Wu, M.; Keel, C.; Yang, B.; Showalter, V.M.; Abood, M.E.; Martin, B.R. Influence of the $\mathrm{n}-1$ alkyl chain length of cannabimimetic indoles upon $\mathrm{cb}(1)$ and $\mathrm{cb}(2)$ receptor binding. Drug Alcohol Depend. 2000, 60, 133-140. [CrossRef] 
75. Huffman, J.W.; Zengin, G.; Wu, M.J.; Lu, J.; Hynd, G.; Bushell, K.; Thompson, A.L.; Bushell, S.; Tartal, C.; Hurst, D.P.; et al. Structure-activity relationships for 1-alkyl-3-(1-naphthoyl)indoles at the cannabinoid $\mathrm{cb}(1)$ and $\mathrm{cb}(2)$ receptors: Steric and electronic effects of naphthoyl substituents. New highly selective $\mathrm{cb}(2)$ receptor agonists. Bioorg. Med. Chem. 2005, 13, 89-112. [CrossRef]

76. Martin, S.J.; Grimwood, P.D.; Morris, R.G. Synaptic plasticity and memory: An evaluation of the hypothesis. Annu. Rev. Neurosci. 2000, 23, 649-711. [CrossRef]

77. Uchiyama, N.; Kikura-Hanajiri, R.; Goda, Y. Identification of a novel cannabimimetic phenylacetylindole, cannabipiperidiethanone, as a designer drug in a herbal product and its affinity for cannabinoid $\mathrm{cb}(1)$ and $\mathrm{cb}(2)$ receptors. Chem. Pharm. Bull. 2011, 59, 1203-1205. [CrossRef]

78. Bito, H.; Deisseroth, K.; Tsien, R.W. Creb phosphorylation and dephosphorylation: A ca(2+)- and stimulus duration-dependent switch for hippocampal gene expression. Cell 1996, 87, 1203-1214. [CrossRef]

79. Rubino, T.; Parolaro, D. Long lasting consequences of cannabis exposure in adolescence. Mol. Cell. Endocrinol. 2008, 286, S108-S113. [CrossRef]

80. Rubino, T.; Sala, M.; Vigano, D.; Braida, D.; Castiglioni, C.; Limonta, V.; Guidali, C.; Realini, N.; Parolaro, D. Cellular mechanisms underlying the anxiolytic effect of low doses of peripheral delta9-tetrahydrocannabinol in rats. Neuropsychopharmacology 2007, 32, 2036-2045. [CrossRef]

81. Maurer, H.H.; Sauer, C.; Theobald, D.S. Toxicokinetics of drugs of abuse: Current knowledge of the isoenzymes involved in the human metabolism of tetrahydrocannabinol, cocaine, heroin, morphine, and codeine. Ther. Drug Monit. 2006, 28, 447-453. [CrossRef]

82. Razdan, R.K.; Vemuri, V.K.; Makriyannis, A.; Huffman, J.W. Cannabinoid Receptor Ligands and Structure-Activity Relationships; Humana Press: Totowa, NJ, USA, 2009; pp. 3-94.

83. Wohlfarth, A.; Scheidweiler, K.B.; Chen, X.; Liu, H.F.; Huestis, M.A. Qualitative confirmation of 9 synthetic cannabinoids and 20 metabolites in human urine using lc-ms/ms and library search. Anal. Chem. 2013, 85, 3730-3738. [CrossRef]

84. Seely, K.A.; Brents, L.K.; Radominska-Pandya, A.; Endres, G.W.; Keyes, G.S.; Moran, J.H.; Prather, P.L. A major glucuronidated metabolite of jwh-018 is a neutral antagonist at cb1 receptors. Chem. Res. Toxicol. 2012, 25, 825-827. [CrossRef]

85. Compton, D.M.; Seeds, M.; Pottash, G.; Gradwohl, B.; Welton, C.; Davids, R. Adolescent exposure of jwh-018 "spice" produces subtle effects on learning and memory performance in adulthood. J. Behav. Brain Sci. 2012, 2, 146-155. [CrossRef]

86. Chin, C.N.; Murphy, J.W.; Huffman, J.W.; Kendall, D.A. The third transmembrane helix of the cannabinoid receptor plays a role in the selectivity of aminoalkylindoles for $\mathrm{cb} 2$, peripheral cannabinoid receptor. J. Pharmacol. Exp. Ther. 1999, 291, 837-844.

87. Irie, T.; Kikura-Hanajiri, R.; Usami, M.; Uchiyama, N.; Goda, Y.; Sekino, Y. Mam-2201, a synthetic cannabinoid drug of abuse, suppresses the synaptic input to cerebellar purkinje cells via activation of presynaptic cb1 receptors. Neuropharmacology 2015, 95, 479-491. [CrossRef]

88. Tomiyama, K.; Funada, M. Cytotoxicity of synthetic cannabinoids found in "spice" products: The role of cannabinoid receptors and the caspase cascade in the ng 108-15 cell line. Toxicol. Lett. 2011, 207, 12-17. [CrossRef]

89. Silva, J.P.; Carmo, H.; Carvalho, F. The synthetic cannabinoid xlr-11 induces in vitro nephrotoxicity by impairment of endocannabinoid-mediated regulation of mitochondrial function homeostasis and triggering of apoptosis. Toxicol. Lett. 2018, 287, 59-69. [CrossRef]

90. Rajasekaran, M.; Brents, L.K.; Franks, L.N.; Moran, J.H.; Prather, P.L. Human metabolites of synthetic cannabinoids jwh-018 and jwh-073 bind with high affinity and act as potent agonists at cannabinoid type-2 receptors. Toxicol. Appl. Pharmacol. 2013, 269, 100-108. [CrossRef]

91. Funada, M.; Takebayashi-Ohsawa, M. Synthetic cannabinoid am2201 induces seizures: Involvement of cannabinoid cb1 receptors and glutamatergic transmission. Toxicol. Appl. Pharmacol. 2018, 338, 1-8. [CrossRef]

92. Koller, V.J.; Auwarter, V.; Grummt, T.; Moosmann, B.; Misik, M.; Knasmuller, S. Investigation of the in vitro toxicological properties of the synthetic cannabimimetic drug cp-47,497-c8. Toxicol. Appl. Pharmacol. 2014, 277, 164-171. [CrossRef] 
93. Patton, A.L.; Seely, K.A.; Yarbrough, A.L.; Fantegrossi, W.; James, L.P.; McCain, K.R.; Fujiwara, R.; Prather, P.L.; Moran, J.H.; Radominska-Pandya, A. Altered metabolism of synthetic cannabinoid jwh-018 by human cytochrome p450 2c9 and variants. Biochem. Biophys. Res. Commun. 2018, 498, 597-602. [CrossRef]

94. Schindler, C.W.; Gramling, B.R.; Justinova, Z.; Thorndike, E.B.; Baumann, M.H. Synthetic cannabinoids found in "spice" products alter body temperature and cardiovascular parameters in conscious male rats. Drug Alcohol Depend. 2017, 179, 387-394. [CrossRef] [PubMed]

(C) 2019 by the authors. Licensee MDPI, Basel, Switzerland. This article is an open access article distributed under the terms and conditions of the Creative Commons Attribution (CC BY) license (http://creativecommons.org/licenses/by/4.0/). 